\title{
Bird forecasting
}

In our restless world, annual bird migrations can provide a transitory opportunity to glimpse beautiful avian visitors (pictured, the migratory Baltimore oriole; Icterus galbula). Birds' journeys are influenced by the local daily weather, making it hard to predict when migrating birds will pass through a particular place on their route. Writing in Science, Van Doren and Horton report a model that forecasts bird migrations (B. M. Van Doren and K. G. Horton Science 361, 1115-1118; 2018).

They created their model (http://birdcast.info) by analysing bird migrations using 23 years of radar data from 143 locations across the United States, and assessing the data on weather conditions for the migrations. Being able to accurately predict an influx of birds might enable temporary measures to be taken to protect these migrants from hazards: for example, by turning off wind turbines. Mary Abraham

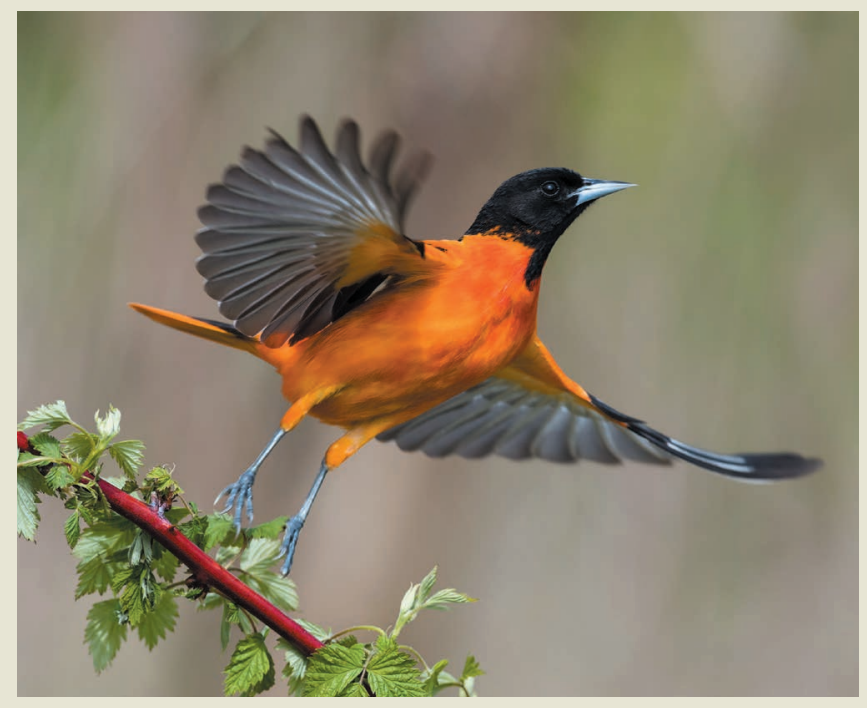

METROLOGY

\section{Timing the action of light on matter}

Photoemission, the ejection of an electron from a material on the absorption of a photon, is one of the fastest processes in nature. An experiment demonstrates how the dynamics of this process can be captured in real time. SEE LETTER P.374

\section{THOMAS FENNEL}

$\mathrm{I}$ t seems natural that light facilitates photosynthesis, enables visual perception and provides the energy source for solar cells. But the underlying light-absorption process is not fully understood. Energy is transferred from the light to electrons in the irradiated material, which can cause electrons to be ejected - a phenomenon known as photoemission. The dependence of the electron ejection on the frequency of the incident light led to Albert Einstein's discovery ${ }^{1}$ that light comes in discrete packets of energy (photons) and sparked the development of quantum mechanics. But how fast can an electron absorb a photon and escape? On page 374 , Ossiander et al. ${ }^{2}$ show how metrology on the attosecond $\left(10^{-18}\right.$ seconds) timescale can help to answer this fundamental question.

It is only in the past decade or so that flashes of light could be generated that are short enough for researchers to directly track the dynamics of photoemission and to obtain timing information on the ejection of electrons ${ }^{3,4}$. This advance has resulted in a vibrant revival of scientific interest in the fundamental physics of photoemission. The timing information contains valuable details about the electronic structure of the target material, many-body effects (the correlated and collective behaviour of many interacting electrons) and the propagation of the electrons after photon absorption.

One of the key instruments used to carry out photoemission measurements is the attosecond streak camera ${ }^{5}$. In experiments based

on this instrument, a material is exposed to an attosecond-duration light pulse that has a frequency corresponding to the extremeultraviolet region of the electromagnetic spectrum. Electrons in the material absorb photons from the pulse and are ejected. These electrons are then accelerated by the electric field of a second light pulse - known as the streaking field - and the final energy of the electrons is measured.

Adjusting the time delay between the two light pulses changes the final electron energy in a well-defined way. This relationship enables a reconstruction of either the time evolution of the streaking field ${ }^{6}$ or the ejection time of the electrons ${ }^{3,4}$, but not both simultaneously. As a result, streaking experiments have been unable to determine absolute photoemission delays time differences between light absorption and electron ejection. Instead, they have provided
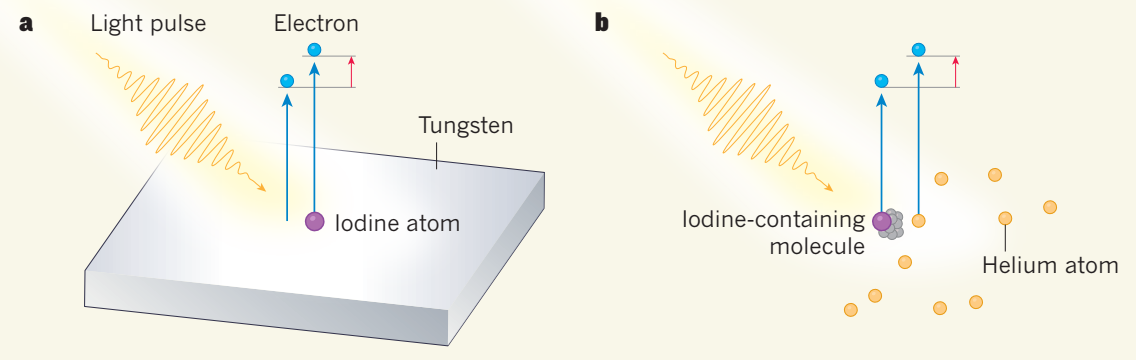

Figure 1 | Measurement of an absolute photoemission delay. Photoemission is the process by which an electron absorbs light and is ejected from a material. Ossiander $\mathrm{et} \mathrm{al} .{ }^{2}$ demonstrate a technique for determining absolute photoemission delays - time differences between light absorption and electron ejection. a, The authors deposited iodine molecules on a clean tungsten surface (for simplicity, a single iodine atom is shown here) and applied an extreme-ultraviolet light pulse to the material. Electrons were ejected (blue arrows) from both the tungsten surface and the iodine atoms. The authors measured the relative delay (red arrow) between these two ejections. b. Ossiander and colleagues then applied the same light pulse to a gaseous mixture of iodine-containing molecules and helium atoms, and measured the relative iodine-helium photoemission delay. Finally, they used the known absolute photoemission delay for helium and the measured relative delays to determine the absolute photoemission delay for the tungsten surface. 